\title{
Concentrated Delivery Schemes Research Focused On Electric Maintenance Materials
}

\author{
Xu Wei ${ }^{1, a}$, Hao Zejun ${ }^{2}$ \\ ${ }^{1}$ Huazhong University of Science and Technology, Wuhan Hubei 430074, China \\ ${ }^{2}$ State Grid Hubei Electric Power Company, Wuhan Hubei 430077, China \\ axwlaina@163.com
}

\begin{abstract}
Keywords: concentrated delivery, electric maintenance materials, long-term average cost, service
\end{abstract} levels

\begin{abstract}
For the characteristics of the demand of the electric maintenance materials is frequent and smaller single-demand, this paper discuss the concentrated delivery mode in vendor-managed inventory (VMI). Two different concentrated delivery schemes have been proposed in the literature for the problem: the time-based scheme and the quantity-based scheme, then we break down the total inventory and distribution costs, and the long-term average cost and average waiting time of the two schemes are given under the condition of the optimal decision. At last, we conduct an empirical analysis combined with the electric maintenance materials distribution of a grid enterprise, the results show that, relative to the real-time replenishment delivery scheme, concentrated delivery schemes have lower cost, and in the two strategies, the quantity-based scheme has lowest cost, but the time-based scheme which takes into account the costs and service levels is the more comprehensive scheme, this scheme guarantee their service levels on the basis of economies of scale.
\end{abstract}

\section{Introduction}

With further promotion of the system of "three sets of five major", the power company proposed new higher level management requirements for management of material distribution. The grid company hopes to introduce competition in all aspects to improve the entire electricity supply chain efficiency and reduce the cost of the electricity supply chain, where the logistics cost as power supply chain management's core has great reform potential. According to incomplete statistics, physical distribution costs account for about $10 \%$ of the total cost of the power companies in the logistics, and the numbers show an increasing trend. Currently, in the construction of power grid supplies intensive management, procurement chain has achieved the centralized purchasing, but in the link of contract compliance and distribution the business is still managed by materials management departments at all levels. Practice has proved that the current management model is not conducive to improving service levels of material supply, the overall control and reducing costs, therefore it is necessary to investigate the optimization of the distribution model to achieve lean management for material distribution. According to maintenance materials' characteristics of smaller single-demand, frequent demand, with a lag and low cost of waiting, this paper introduces concentrated delivery schemes. In this way, the provincial power company supplies deployment center no longer passively delivers and replenishes in accordance with the needs of maintenance materials of the downstream city supply center, but according to the needs for materials under the jurisdiction of numerous city supply centers, the provincial power company supplies deployment center schedule a more rational way of delivery, in the way the supplies deployment center can meet the needs of the city supply centers, while making their inventory management and replenishment policy more rational, thus reducing the overall cost of the supply chain.

The centralized delivery schemes are studied in vendor-managed inventory [1]. In [1], bulk shipments and instant delivery were compared and researched. In [2], the quantity-based scheme and mixed delivery scheme were proposed on the basis of the time-based scheme, and optimal 
delivery time, delivery times and the delivery numbers were proposed on the basis of the optimal cost. In [3], the optimal decisions of the time-based scheme and the quantity-based scheme were studied under the consideration of the lead time. In [5], the optimal choice for centralized delivery was studied under the consideration of service levels. Different from these papers focused on either the costs or service levels, this paper gives the optional choice of centralized delivery schemes by taking into account the costs, service levels and complete ordering costs and delivery costs to make it closer to the real situation.

\section{Related concepts and definitions}

The meaning of centralized delivery and two centralized delivery schemes. The centralized delivery gathers a lot of small batches ship to fewer shipments of larger load in order to reduce the delivery cost. In this management model, the provincial supplies deployment center determines the delivery time and delivery number for the city supply center. The provincial supplies deployment center schedule a more rational way of delivery under the consideration of the time and the delivery destination of the orders of the city supply center, then determine their own inventory replenishment methods based on the delivery way. In this delivery method, two schemes are commonly used in industry: the time-based scheme and the quantity-based scheme. Under the time-based scheme, a shipment is dispatched periodically whereas under the quantity-based scheme, a shipment is made only when a certain quantity of outstanding demands is accumulated. A time-based scheme thus assures each demand is shipped within a predetermined time frame provided that there is sufficient stock on-hand.

Problem description. The centralized delivery model studied by this paper is improved on the basic of the VMI integrated delivery model. There are suppliers, the provincial supplies deployment center and the downstream city supply centers in the model. R1, R2, R3 distributed within a given region. Assume demand for a city supply center to reach the provincial supplies deployment center is a Poisson demand process of rate $\lambda$. Within each delivery cycle(the time from you start to make decisions about the shipment to the next time to make decisions about the shipment), the provincial supplies deployment center will put together the demand orders to form a large number of shipments. The inventory status of the provincial materials warehouse after delivery cycle is current inventory minus the accumulated demand of the previous delivery period. Once the provincial supplies deployment center make the decision about a delivery, all current demands (ie the accumulated demand of current delivery cycle) must be met. If the provincial supplies deployment center make the decision about a delivery, the current inventory is not sufficient to meet all the needs, the provincial materials warehouse should immediately supplement inventory to meet demand. To simplify the analysis and comparison we assume zero lead time for replenishment orders. Then an ${ }^{(s, S)}$ policy with $\mathrm{s}=-1$ and $S \geq 0$ is applied for replenishing the inventory in the provincial materials warehouse. The lead time is zero, so by choosing $s=-1$ we can guarantee that the demand during a period can always be dispatched at the end of the period. After dispatching the demand from the previous period, the inventory on hand is always in the interval ${ }^{[0, S]}$.

The total cost includes four parts: the replenishment cost, inventory holding costs, customer waiting cost and shipping cost.

\section{Centralized delivery schemes}

Relevant parameters and variables. We introduce the following notations in the model:

$\lambda=$ demand intensity, $A_{R}=$ a fixed cost of replenishing inventory,

$U_{R}=$ replenishing inventory cost per unit, $A_{D}=$ a fixed cost of dispatching,

$U_{D}=$ dispatching cost per unit, $w=$ customer waiting cost per unit and time unit,

$h=$ holding cost per unit and time unit, $S_{n}=$ time the n-th demand arrival,

$N(t)=$ demand of $t$ unit time, ${ }^{I(t)}=$ inventory status on time T, $Q=$ target inventory level, 
$N_{\mathrm{j}}=$ delivery number of the $\mathrm{j}$-th delivery cycle, $\mathrm{j}=1,2, C=$ total costs per time unit,

$T_{\mathrm{j}}=$ time length of the $\mathrm{j}$-th delivery cycle, $\mathrm{j}=1,2, K=$ delivery times of a replenishing cycle,

$C=\frac{E[\text { the total cost of each replenishment cycle }]}{E[\text { the length of each replenishment cycle }]}$.

The time-based scheme. Under the time-based scheme, the provincial supplies deployment center dispatch a shipment per time T periodically. A time-based scheme thus assures each demand is shipped within the time $\mathrm{T}$. Therefore, the maximum delay time of delivery, the holding time orders, equal to the length of a delivery cycle.

Set $C(Q, T)$ be the total costs per time unit of the time-based scheme model, so the model problem is transformed into

$\min C(Q, T) \quad$ s. to $Q \geq 0, T \geq 0$

Now the problem is to calculate $C(Q, T)$. Because the integrated delivery model is updated once per unit of time T, so

$E[$ the length of each replenishment cycle $]=E[K] T$

As mentioned above, the total cost includes the replenishment cost, inventory holding costs, customer waiting cost and shipping cost. The cost of these four parts is calculated as follows:

(1) Expected replenishment cost of each replenishment cycle

$E_{1}=A_{R}+U_{R}\left(\sum_{j=1}^{K} N_{j}\right)=A_{R}+K \lambda T U_{R}$

(2) Expected delivery cost of each replenishment cycle

$E_{2}=K A_{D}+U_{D}\left(\sum_{j=1}^{K} N_{j}\right)=K A_{D}+K \lambda T U_{D}$

(3) According to the characteristics of the time-based scheme we can obtain

$$
I(t)=\left\{\begin{array}{c}
Q, \quad Q \in t \leq T \\
Q-N_{1}, T \leq t \leq 2 T \\
\vdots \\
Q-\sum_{\mathrm{j}=1}^{K} N_{j},(K-1) T \leq t \leq K T
\end{array}\right.
$$

Expected inventory holding costs of each replenishment cycle can be described as follow:

$E_{3}=h\left\{Q T+\left(Q-N_{1}\right) T+\cdots\left(Q-\sum_{j=1}^{K} N_{j}\right) T\right\}=h\left\{\frac{[Q T+(\lambda T-1) T]}{2} K\right\}$

(4) First, we only consider the customer waiting costs of an integrated delivery cycle, $E$ [customer waiting costs of each integrated delivery cycle]

$=w E\left[\left(T-S_{1}\right)+\left(T-S_{2}\right)+\cdots+\left(T-S_{N_{j}}\right)\right]=w \frac{T-\frac{1}{\lambda}+0}{2} \lambda T=w \frac{\lambda T^{2}-T}{2}$

So the customer waiting costs of each replenishment cycle can be expressed as

$E_{4}=K E[$ customer waiting costs of each integrated delivery cycle $]=\mathrm{wK} \frac{\lambda T^{2}-T}{2}$

Expect delivery times of each replenishment cycle is $E[K]=\frac{Q+1}{\lambda T}$

By the formula (1), (2), (3), (4), (5), (6), we can obtain

$C(Q, T)=\frac{A_{R} \lambda}{Q+1}+\frac{h Q}{2}+\frac{h \lambda T}{2}-\frac{h}{2}+\frac{A_{D}}{T}+\frac{\mathrm{w} \lambda T}{2}-\frac{w}{2}+(Q+1)\left(U_{R}+U_{D}\right)$

Now we show how $C(Q, T)$ can be optimized efficiently with respect to $Q$ and $T$. Since $C(Q, T)$ does not contain the cross-term of $Q$ and $T$, the optimal solution $\left(Q^{*}, T^{*}\right)$ must satisfy the following equations: 


$$
\left\{\begin{array}{c}
\frac{\partial C(Q, T)}{\partial Q}=0 \\
\frac{\partial C(Q, T)}{\partial T}=0 \\
Q \geq 0 \\
T \geq 0
\end{array}\right.
$$

According to the equations above, taking into account the fact that $Q$ must be non-negative integers, we can get the optimal solution.

$$
\left\{\begin{array}{c}
Q^{*}=\max \left\{\left[\sqrt{\frac{2 A_{R} \lambda}{h+2\left(U_{R}+U_{D}\right)}}-1\right], 0\right\} \\
T^{*}=\sqrt{\frac{2 A_{D}}{(h+w) \lambda}}
\end{array}\right.
$$

Under the time-based scheme, the optimal cost solution is $\left(Q^{*}, T^{*}\right)$. In this case the average material waiting time of the downstream city supply centers is $\frac{\lambda T-1}{2 \lambda}$, the total costs per time unit can be expressed as

$$
C\left(Q^{*}, T^{*}\right)=\frac{A_{R} \lambda}{Q^{*}+1}+\frac{h Q^{*}}{2}+\frac{h \lambda T^{*}}{2}-\frac{h}{2}+\frac{A_{D}}{T^{*}}+\frac{\mathrm{w} \lambda T^{*}}{2}-\frac{w}{2}+\left(Q^{*}+1\right)\left(U_{R}+U_{D}\right)
$$

The quantity-based scheme .Under the quantity-based scheme, provincial materials warehouse makes a shipment when a certain quantity of outstanding demands is accumulated. The number of each shipment is $Q_{e}$. Therefore, under this scheme the problem can be transformed into

$$
\min \quad C\left(Q_{e}, K\right) \quad \text { s. to } \quad Q_{e} \geq 1, K \geq 1
$$

By calculation like the above, we can obtain

$C\left(Q_{e}, K\right)=\frac{A_{R} \lambda}{K Q_{e}}+\frac{h(K-1) Q_{e}}{2}+\frac{A_{D} \lambda}{Q_{e}}+\frac{w\left(Q_{e}-1\right)}{2}+\lambda\left(U_{D}+U_{R}\right)$

Now we show how $C\left(Q_{e}, K\right)$ can be optimized efficiently with respect to $Q_{e}$ and $K$. Since $C\left(Q_{e}, K\right)$ contains the cross-term of $Q_{e}$ and $K, Q_{e}$ and $K$ need to be treated as continuous variables for the sake of the optimal cost solution.

$\mathrm{By}\left\{\begin{array}{l}\frac{\partial C}{\partial K}=0 \\ \frac{\partial C}{\partial Q_{\mathrm{e}}}=0\end{array}\right.$, the stagnation can be obtained $\left\{\begin{array}{c}K^{*}=\sqrt{\frac{A_{R}(w-h)}{h A_{D}}} \\ Q_{e}^{*}=\sqrt{\frac{2 \lambda A_{D}}{w-h}}\end{array}\right.$

$$
\text { Obtained from (11) }\left\{\begin{array}{c}
\frac{\partial^{2} C}{\partial K^{2}}=\frac{2 A_{R} \lambda}{Q_{e} K^{3}}>0 \\
\frac{\partial^{2} C}{\partial Q_{e}^{2}}=\frac{2 \lambda}{Q_{e}^{3}}\left(\frac{A_{R}}{K}+A_{D}\right)>0 \\
\frac{\partial^{2} C}{\partial Q_{e} \partial K}=\frac{A_{R} \lambda}{Q_{e}^{2} K^{2}}+\frac{h}{2}
\end{array}\right.
$$

Then $\frac{\partial^{2} C}{\partial K^{2}} \cdot \frac{\partial^{2} C}{\partial Q_{e}^{2}}-\left(\frac{\partial^{2} C}{\partial Q_{e} \partial K}\right)^{2}=\frac{3 \lambda^{2} A_{R}^{2}}{Q_{e}^{4} K^{4}}+\frac{4 \lambda^{2} A_{R} A_{D}}{Q_{e}^{4} K^{3}}-\frac{h^{2}}{4}-\frac{h \lambda A_{R}}{Q_{e}^{2} K^{2}}$

Since we cannot determine the positive or negative of the formula (14), we need to determine the size according to the actual value of the parameter. Then we can find the optimal decision $\left(Q_{e}^{*}, K^{*}\right)$ of the model and the optimal solution $C\left(Q_{e}^{*}, K^{*}\right)$. In this case the average material waiting time of the downstream city supply centers is $\frac{Q_{e}^{*}-1}{2 \lambda}$. 


\section{Numerical examples and comparison}

We select the demand for cables in an area of Hubei province as an example. Assure the demand of the seven county supplies center in the area are $\lambda_{\mathrm{i}}(i=1,2,3 \ldots 7)$ (unit: Tie)( 30 days for a unit of time). By examining distribution costs and the demands of the area of Hubei Province from 2011 to 2013, we can get the parameter values in the model as follows.

$A_{D}=200, U_{D}=15, A_{R}=250, U_{R}=15, w=60, h=15$;

By fitting the demands of the seven county supplies center, we get the demands parameter values. $\lambda_{1}=2, \lambda_{2}=2, \lambda_{3}=1, \lambda_{4}=3, \lambda_{5}=2, \lambda_{6}=4, \lambda_{7}=3$

(1) Without centralized delivery scheme, the expected total costs per time unit of the real-time replenishment delivery scheme can be calculated as follows.

$C=200 \times 7+17 \times 15+250 \times 7+17 \times 15=3660$. Then the waiting time is 0 .

(2) Under the time-based scheme, we can obtain the optimal cost solution thought the above formulas.

$$
\begin{gathered}
Q^{*}=\max \left\{\left[\sqrt{\frac{2 A_{R} \lambda}{h+2\left(U_{R}+U_{D}\right)}}-1\right], 0\right\}=[9.6458]=10 \\
T^{*}=\sqrt{\frac{2 A_{D}}{(h+w) \lambda}}=0.5601
\end{gathered},
$$

Then the delivery time of optimal decision should be $0.5601 \times 30=16.80=17$ days, target inventory level should be 10 ties. In this case total costs per time unit are $C(Q, T)=1468.0065$, and the average waiting time is $0.2506 \times 30=7.52$ days.

(3) Under the quantity-based scheme, thought the above data we can obtain:

$\frac{\partial^{2} C}{\partial K^{2}} \cdot \frac{\partial^{2} C}{\partial Q_{e}^{2}}-\left(\frac{\partial^{2} C}{\partial Q_{e} \partial K}\right)^{2}=\frac{3 \lambda^{2} A_{R}^{2}}{Q_{e}^{4} K^{4}}+\frac{4 \lambda^{2} A_{R} A_{D}}{Q_{e}^{4} K^{3}}-\frac{h^{2}}{4}-\frac{h \lambda A_{R}}{Q_{e}^{2} K^{2}}>0$

So we can obtain the optimal cost solution as follows.

$\left\{\begin{array}{c}K^{*}=\max \left\{\left[\sqrt{\frac{A_{R}(w-h)}{h A_{D}}}\right], 1\right\}=[1.9365]=2 \\ Q_{e}^{*}=\max \left\{\left[\sqrt{\frac{2 \lambda A_{D}}{w-h}}\right], 1\right\}=[12.2927]=13\end{array}\right.$

Then the delivery times of optimal decision should be 2 , the delivery shipments should be 13 ties. In this case total costs per time unit are $C\left(Q_{e}^{*}, K^{*}\right)=1392.5$, and the average waiting time is $0.3529 \times 30=10.59$ days.

Table 1 describes the comparison of three delivery schemes.

Table 1: Expected cost and service levels of the three delivery schemes

\begin{tabular}{ccc}
\hline Delivery Scheme & $\begin{array}{c}\text { Long-Term Average } \\
\text { Costs(/Y) }\end{array}$ & $\begin{array}{c}\text { Average Waiting } \\
\text { Time(/Day) }\end{array}$ \\
\hline Real-Time Delivery Scheme & 3660.0000 & 0 \\
The Time-Based Scheme & 1468.0065 & 7.52 \\
The Quantity-Based Scheme & 1392.5000 & 10.59
\end{tabular}

As can be seen from the table 1, compared to the real-time replenishment delivery scheme, centralized delivery schemes significantly reduce the cost of the model, but they also pay the price decline in the quality of service. In the two centralized delivery schemes, the cost of the quantity-based scheme is minimized in the optimal decision. And the number of each delivery is a fixed value, which can ensure acquire the scale economies, but the service level is the lowest. The time-based scheme guarantees part of scale economies but also takes into account the quality of service, to ensure that each demand can be met within a pre-determined time.

In order to verify the above conclusions are incorporated in the general case, the paper proceeds 
further numerical experiments. In the case of other parameters unchanged, the sensitivity for the total demand of the two centralized delivery schemes and the real-time replenishment delivery scheme are proposed. The optimal solution and service levels of the three schemes of different total demands are expressed as Table 2.

Table 2 Impact of changes in total demand for the optimal solution

\begin{tabular}{ccccccc}
\hline & \multicolumn{2}{c}{$\lambda=15$} & \multicolumn{2}{c}{$\lambda=17$} & \multicolumn{2}{c}{$\lambda=20$} \\
\cline { 2 - 7 } Delivery Scheme & $\begin{array}{l}\text { Long-Term } \\
\text { Average } \\
\text { Costs(/Y) }\end{array}$ & $\begin{array}{l}\text { Average } \\
\text { Waiting } \\
\text { Time(/Day) }\end{array}$ & $\begin{array}{c}\text { Long-Term } \\
\text { Average } \\
\text { Costs(/Y) }\end{array}$ & $\begin{array}{l}\text { Average } \\
\text { Waiting } \\
\text { Time(/Day) }\end{array}$ & $\begin{array}{l}\text { Long-Term } \\
\text { Average } \\
\text { Costs(/Y) }\end{array}$ & $\begin{array}{l}\text { Average } \\
\text { Waiting } \\
\text { Time(/Day) }\end{array}$ \\
\hline $\begin{array}{c}\text { Real-Time Delivery } \\
\text { Scheme }\end{array}$ & 3600.00 & 0 & 3660.00 & 0 & 3750.00 & 0 \\
$\begin{array}{c}\text { The Time-Based } \\
\text { Scheme }\end{array}$ & 1375.82 & 7.9445 & 1468.01 & 7.52 & 1596.26 & 7 \\
$\begin{array}{c}\text { The Quantity-Based } \\
\text { Scheme }\end{array}$ & 1276.25 & 11 & 1392.50 & 10.59 & 1559.29 & 9.75
\end{tabular}

From table 2, the results well validate the above conclusion. Compared to the real-time replenishment delivery scheme, centralized delivery schemes significantly reduce the cost of the model. The quantity-based scheme and the time-based scheme have each advantages and disadvantages. The quantity-based scheme can minimize the costs, and the time-based scheme is more comprehensive consideration of the costs and service levels.

\section{Conclusions}

In this paper, the strengths and weaknesses of two centralized delivery model and real-time delivery model are proposed by comparing their optimal delivery numbers, delivery times, optimal cost, and service level. The paper can provide theoretical basis for decision-makers of materials distribution which can make decisions about materials distribution under the consideration of the actual situation. The grid company can implement differentiated shipping methods for different materials. The service levels of the two centralized delivery schemes are analyzed at the situation of optimal cost. Objective function can also be established by weighting cost and service level, and then the optimal solution and optimal decision will be obtained. In addition, for different regions, we can predict their demands based on their previous data so as to give more maneuverability for the provincial supplies deployment center.

\section{References}

[1] S. Viswanathan. European Journal of Operational Research, 1998, Vol.105 (1), pp.38-42.

[2] Liu Liwen, Yuan Jiarui. Chinese Journal of Management Science, 2003, 11(5): 31-36. (In Chinese)

[3] Wang Zhengguo. Huazhong University of Science and Technology, 2006. (In Chinese)

[4] Du Shaofu. Dissertation of University of Science and Technology of China, 2007. (In Chinese)

[5] Ji Shoufeng, Fu Chengjun, Huang Xiaoyuan. Journal of Northeastern University ( Natural Science), 2006, 27(10): 1169-1172.

[6] Axsäter S. Management Science, 2001, 47(9): 1306-1310.

[7] Chen F Y, Wang T, Xu T Z. Annals of Operations Research, 2005, 135(1): 197-210. 